\title{
Estudio Cinético y de Superficie de Respuesta para la Rehidratación de Zanahorias (Daucus carota) Liofilizadas
}

\author{
María L. Zambrano, Deborah B. Rodríguez y Alfredo Álvarez \\ Universidad Nacional Autónoma de México, Facultad de Estudios Superiores Cuautitlán, \\ Departamento de Ingeniería y Tecnología, Av. 1ro. de Mayo s/n, Col. Atlanta, Cuautitlán Izcalli, \\ 54740 Estado de México-México (luz.zambrano@correo.unam.mx; alfac@servidor.unam.mx)
}

\begin{abstract}
Resumen
Se estudio el efecto de la temperatura sobre la cinética, el coeficiente de difusión y la adición de ácido cítrico y cloruro de sodio sobre la rehidratación de zanahorias (Daucus carota). Se aplicó un diseño de superficie de respuesta con 4 factores y 3 puntos centrales. No se encontró diferencia significativa $(\alpha=0.05)$ a temperaturas de 70 y $93^{\circ} \mathrm{C}$ con constante cinética promedio de $0.42 \mathrm{~min}^{-1}$, con diferencias entre los coeficientes de difusión entre 6.1 a $3.18 \times 10^{-9} \mathrm{~m}^{2} / \mathrm{s}$. Las variables de mayor influencia sobre la rehidratación son el contenido de ácido cítrico, la temperatura y la concentración de ácido cítrico, a los niveles estudiados. Las condiciones de mayor rehidratación para las muestras de zanahoria liofilizada fueron obtenidas para una concentración de $0.636 \mathrm{~g} / 100 \mathrm{ml}$ ácido, con un óptimo de $7 \mathrm{~kg}$ de agua/kg de sólido seco, de acuerdo al modelo de superficie de respuesta obtenido. El valor promedio fue de $6.65 \mathrm{~kg}$ de agua $/ \mathrm{kg}$ de sólido seco.
\end{abstract}

Palabras clave: Daucus carota, rehidratación, cinética de primer orden, superficie de respuesta

\section{Kinetic Study and Surface Response Analysis on the Rehydration of Frozen-dried Carrot (Daucus carota)}

\begin{abstract}
In this work the effect of temperature on the kinetics, the diffusion coefficient sand the addition of citric acid and sodium chloride on the rehydration of frozen-dried carrots (Daucus carota) have been studied. The model response surface was 4 factors with 3 central points. For 70 and $93{ }^{\circ} \mathrm{C}$, no significant differences $(\alpha=0.05)$ for the kinetic constant with mean of $0.42 \mathrm{~min}^{-1}$. Important differences were found between diffusion coefficients, from 6.1 to $3.18 \times 10^{-9} \mathrm{~m}^{2} / \mathrm{s}$ for 70 y $93^{\circ} \mathrm{C}$. The variables with higher influence on rehydration were the content of citric acid, citric acid concentration and temperature, at the studied levels. The best condition for rehydration of carrot frozen-dried was obtained for $0.636 \mathrm{~g} / 100 \mathrm{ml}$ acid concentration with $7 \mathrm{~kg}$ of water $/ \mathrm{kg}$ dry solid, using the surface model. The mean value was $6.65 \mathrm{~kg}$ of water $/ \mathrm{kg}$ dry solid.
\end{abstract}

Keywords: Daucus carota, rehydration, first order kinetic, model response surface 


\section{INTRODUCCIÓN}

Desde tiempos inmemoriales los procesos de deshidratación han sido de interés en la conservación de alimentos, actualmente se han desarrollado tecnologías que se enfocan en la conservación de las características del producto con el fin de complacer los criterios de calidad del consumidor. Con la eliminación de agua por liofilización se pretende disminuir los daños celulares a los que se ve expuesto un producto por efecto de la deshidratación, de tal manera que al rehidratarse bajo las condiciones de uso tenga las características esperadas (Krokida y Marinos, 2002).

En México las zanahorias variedad (Daucus carota L.), son de amplia producción en la parte central del país y consumidas a nivel nacional y mundial, en una variedad de opciones de procesamiento para su posterior utilización. Una de las formas de trasformación es la deshidratación de producto para ser utilizada como materia prima de otros productos como son sopas instantáneas, alimentos precocinados (fast food) para su calentamiento en microondas acompañados de carne y otros ingredientes, o bien cereales y sopas pre-cocidas como arroz con vegetales, arroz a la mexicana, pastas, tallarines, etc.

Es durante su consumo donde la rehidratación de un producto refleja el efecto de otras variables involucradas en la transformación previa para su conservación. De esas variables, en México existe un gran impacto en la utilización de ácido cítrico (componente principal del limón mexicano) que en combinación con el $\mathrm{NaCl}$ afectaran propiedades importantes en las características de un alimento, en este sentido, existen pocas investigaciones enfocadas a la evaluación del efecto de la adición de otros solutos, gran parte de las que se han realizado evalúan la variación del agua absorbida durante la rehidratación como un reflejo del cambio de calidad del producto por efecto de las condiciones de previas al procesamiento, elección del método y condiciones de secado, así como el almacenamiento posterior de los vegetales deshidratados. (Krokida et al., 2002; Rastogi et al., 2004).

Para contrastar los resultados obtenidos se han empleado diseños experimentales de superficie de respuesta (MSR), factoriales y pruebas de hipótesis (Mudar et al., 1989; Madamba, 2002; Mastrocola et al., 1998). Esta metodología pretende explorar y modelar estadísticamente la relación que existe entre las variables independientes y las de respuesta para posteriormente encontrar los niveles o valores de las variables independientes que logren los valores de respuesta deseados. Para ello, es necesario que la variable(s) de respuesta que se desea analizar se encuentre adecuadamente establecida. Razón por la que en este estudio se debe considerar la forma en que se evaluará la cinética de rehidratación de un producto sometido a liofilización, con la finalidad de tener la menor variabilidad posible en las muestras a tratar, En este sentido se han publicado estudios sobre diferentes productos, entre los que se encuentra la zanahoria y otros vegetales (Marabi et al., 2004; Wang y Xi, 2005), de estos, se tomarán algunos de los criterios para el tratamiento de los resultados experimentales.

Debido a la necesidad de evaluar el efecto de la adición de $\mathrm{NaCl}$ y ácido cítrico a los vegetales deshidratados incorporados a otros productos como las sopas instantáneas, los objetivos de este trabajo fueron: obtener las constantes cinéticas y coeficientes de difusión de agua y evaluar el efecto de la adición de $\mathrm{NaCl}$ y ácido cítrico durante la rehidratación de zanahorias liofilizadas, con el fin de establecer las condiciones optimas mediante un diseño de superficie de respuesta (MSR).

\section{MATERIALES Y MÉTODOS}

\section{Materia prima}

Las zanahorias variedad Dacus carota L., fueron adquiridas, durante el ciclo de primavera 2005, seleccionándose por tamaño con valores promedios de $3.5 \mathrm{~cm}$ de diámetro superior y $9 \mathrm{~cm}$ de longitud. Se lavaron, pelaron y después cortadas en placas de $4 \mathrm{~mm}$ espesor, hasta la parte central de la zanahoria, despreciándose el resto del producto. El contenido inicial de humedad en las zanahorias fue determinado por quintuplicado en una estufa a vacío a $70 \pm 2^{\circ} \mathrm{C}$ por 5 horas (AOAC, 2005). Previo a la liofilización, las zanahorias se sometieron a congelación utilizando un sistema didáctico de congelación por lotes, con una temperatura del medio de $-20^{\circ} \mathrm{C}$, durante 5 horas. 


\section{Secado por congelación a vacío}

Las muestras congeladas se liofilizaron utilizando un equipo de liofilización didáctico marca Educational Engineering S.A. N.V que cuenta con un sistema de producción de frío por compresión mecánica, durante la experimentación no fue utilizada la placa calefactora y la presión absoluta en la cámara fue de $0.0197 \mathrm{~atm}$, el proceso se llevo a cabo durante 4 horas, verificándose al final el porcentaje de humedad de las muestras.

\section{Rehidratación}

Para establecer el método de rehidratación se tomaron en cuenta las siguientes referencias: Método 88-04 de A.A.C.C. (1983); Propiedades funcionales y metodología para su evaluación en fibra dietética" (Zambrano y Gallardo, 2001; Femenia et al., 2000). Las pruebas se realizaron con $2.5 \mathrm{~g}$ de zanahoria, que se rehidrataron en cajas de petri de $10 \mathrm{~cm}$ de diámetro, adicionándoles $25 \mathrm{ml}$ de agua destilada o solución con concentraciones de $\mathrm{NaCl} 1.25$ y $2.5 \mathrm{~g} / 100 \mathrm{ml}$ ó ácido cítrico de 0.375 y $0.75 \mathrm{~g} / 100 \mathrm{ml}$. Los cambios en peso durante la rehidratación se registraron cada 10 minutos, en un intervalo de 10 a 120 minutos.

\section{Cinética de rehidratación}

La constante de velocidad cinética para la rehidratación de zanahorias se determinó tomando la relación de rehidratación $(\mathrm{Mr})$ en función al peso ganado por la muestra con respecto al peso seco.

$-d X / d t=k(X-X e)$

Donde $X$, representa el contenido de humedad de las zanahorias durante la rehidratación (kg de agua/kg de sólido seco), Xe es el contenido de humedad en el equilibrio, $k$ es la velocidad de rehidratación en $\min ^{-1}$, $t$ el tiempo de rehidratación (min), la velocidad de rehidratación esta representada por la pendiente de la curva de acuerdo con la integración de la ecuación (1).

$X=X e-(X e-X i) e^{-k t}$

\section{Coeficientes de difusión del agua}

Para determinar el coeficiente de difusión de agua en zahorias, se consideraron a las muestras como placas infinitas. La solución a la segunda ley de Fick para la difusión en una placa infinita de lados $2 a$, 2b y 2c resulta de la ecuación para la transferencia de agua (Crank, 1975):

$M_{r}=\frac{\left(m_{t}-m_{\infty}\right)}{\left(m_{\circ}-m_{\infty}\right)}=\sum_{n=1}^{\infty} \operatorname{Cn} \exp \left[-D_{e w} t q_{n}^{2}\left[\frac{1}{A^{2}}\right]\right]$

Donde: $m_{t}$ representa el agua absorbida al tiempo $(\mathrm{t}), m_{-}=$agua absorbida en el equilibrio, $m_{\mathrm{o}}=$ contenido de humedad inicial, todos expresados en base seca como $\mathrm{kg}$ de agua/kg de sólido seco, $D_{\text {ew }}$, es la difusividad efectiva de agua en $\mathrm{m}^{2} / \mathrm{s}$, C es la constante de proporcionalidad, $t$ el tiempo en minutos, $q_{\underline{n}}$, la relación de agua/producto, $\mathrm{n}$ las veces que se considera la variación $\mathrm{q}$ hasta el equilibrio y $A$ el área de la placa en $\mathrm{m}^{2}$.

Tabla 1: Nivel de los factores.

\begin{tabular}{|c|c|c|}
\hline Factor & Límite inferior & Límite superior \\
\hline Ác.cítrico $(\mathrm{g} / 100 \mathrm{ml})$ & 0 & 0.750 \\
\hline $\mathrm{NaCl}(\mathrm{g} / 100 \mathrm{ml})$ & 0 & 0.25 \\
\hline Temperatura & 50 & 100 \\
\hline Tiempo (min) & 5 & 15 \\
\hline
\end{tabular}




\section{Diseño Experimental.}

Se seleccionaron como variables del proceso de rehidratación de zanahoria, la temperatura del agua, el tiempo de rehidratación, la concentración de $\mathrm{NaCl}$ y ácido cítrico. Evaluando como variable de respuesta el tiempo de rehidratación, parámetro de mayor relevancia cuando el producto es utilizado para la elaboración de sopas instantáneas o bien productos listos para consumo. Todas las mediciones se realizaron por triplicado y con un muestreo aleatorio.

Puesto que la rehidratación de productos alimenticios tiene variaciones de comportamiento debidos a la estructura del material, se seleccionó un diseño de superficie de respuesta tipo Box-Behnken, con 4 factores, 3 puntos centrales y 3 bloques, los niveles de los factores son mostrados en la Tabla 1. Los bloques se plantearon con la finalidad de disminuir el error debido a los tratamientos. El análisis de resultados se realizó auxiliándose del software para análisis estadísticos MINITAB 14, obteniéndose de este el análisis de varianza y el ajuste a comportamiento lineal y/o cuadrático.

\section{RESULTADOS Y DISCUSIÓN}

\section{Cinética de Rehidratación}

En la Figura 1, se muestra el comportamiento cinético de rehidratación, de donde se tiene que existe una diferencia importante entre el equilibrio alcanzado por las muestras a diferentes temperaturas, ya que en función a la $M_{r}$, se presenta el equilibrio en $5.40 \mathrm{~kg}$ de agua/ $\mathrm{kg}$ de s.s., mientras que la de 75 ${ }^{\circ} \mathrm{C}$ lo alcanza a 6.31 y a $6.65 \mathrm{~kg}$ de agua $/ \mathrm{kg}$ de s.s. la de $93^{\circ} \mathrm{C}$, lo que implica que la menor velocidad se tiene a $50^{\circ} \mathrm{C}$, resultado esperado en relación a los comportamientos cinéticos. Además, en relación con otros estudios realizados y al menor daño celular en las muestras liofilizadas en este trabajo, permiten absorciones mayores que las reportadas para secado convectivo en zanahoria, ya que de acuerdo a lo reportado por Rastogi y Raghavarao (2004), quienes obtuvieron absorciones de $4 \mathrm{~kg}$ agua $/ \mathrm{kg}$ s.s. al realizar la rehidratación de zanahorias a $25^{\circ} \mathrm{C}$ durante cinco horas. Mientras que, Krokida et al. (2003), obtuvieron absorciones de entre $1.9,3.3$ y $5.3 \mathrm{~kg}$ de agua/kg de s.s. al realizar la cinética de rehidratación de zanahorias a 40,60 y $80^{\circ} \mathrm{C}$ respectivamente.

En la tabla 2 Se observan las constantes cinéticas de velocidad de absorción de agua (k), a las diferentes temperaturas, considerando el periodo de velocidad ascendente hasta los 60 minutos, después de este tiempo la absorción de agua permanece prácticamente constante, la $k$ varia considerablemente, por lo que la absorción de agua se facilita a mayor temperatura, aunque la más alta, se obtiene a $75^{\circ} \mathrm{C}$, siendo $19.25 \%$ mayor que la $k$ correspondiente a los $93^{\circ} \mathrm{C}$. Aunque con un nivel de confianza del $95 \%$ se logra establecer que no existe diferencia significativa entre los tratamientos, siendo estos atribuibles las variaciones a los cambios estructurales de la pared celular de las zanahorias en relación al método de eliminación de agua (liofilización). También se muestran los coeficientes de difusión de agua $\left(D_{e w}\right)$, al igual que $k$, la mayor resistencia a la difusión de agua se presenta a los $50^{\circ} \mathrm{C}$ y la menor a los $93^{\circ} \mathrm{C}$, con un valor promedio de $3.18 \times 10^{-9} \mathrm{~m}^{2} / \mathrm{s}$, lo que se contrapone un poco con respecto al comportamiento de la $k$; sin embargo, es necesario resaltar que k corresponde a una correlación cinética y no considera las características geométricas del producto, ni el sentido en que se lleva la difusión, además $D_{\text {ew }}$, es útil para explicar el proceso de transferencia de masa que se lleva a cabo; en este sentido, Lee et al., (2006) reportan valores de $3.08 \times 10^{-7}$ a $9.78 \times 10^{-9} \mathrm{~m}^{2} / \mathrm{s}$, lo que implica que en el caso estudiado y a pesar de que se encuentra entre los valores reportados, la composición de la zanahoria con un alto contenido de lignina contribuye en el comportamiento ante la movilidad de agua.

\section{Superficie de respuesta}

En la tabla 3, se muestran los resultados de la prueba t para los coeficientes de regresión, realizada con $\alpha=0.05$, considerando el ajuste a un modelo cuadrático, mostrándose también el valor de $p$ para estos, lo que implica la importancia de los coeficientes en el modelo. Los tratamientos por bloques, contribuyeron a darle robustez al modelo obtenido, ya que el valor de $\mathrm{R}^{2}$ para el modelo mostró un ajuste del $92 \%$. Los valores de p, que representan el área de una distribución $t$, en donde es probable se encuentren los resultados, justifican que los factores temperatura y tiempo no tienen 
efecto en el comportamiento lineal de la superficie de respuesta; es decir, existe independencia entre estas variables, aunque cabe mencionar que las interacciones de las variables si tienen un efecto significativo sobre el comportamiento de rehidratación de las muestras de zanahoria.

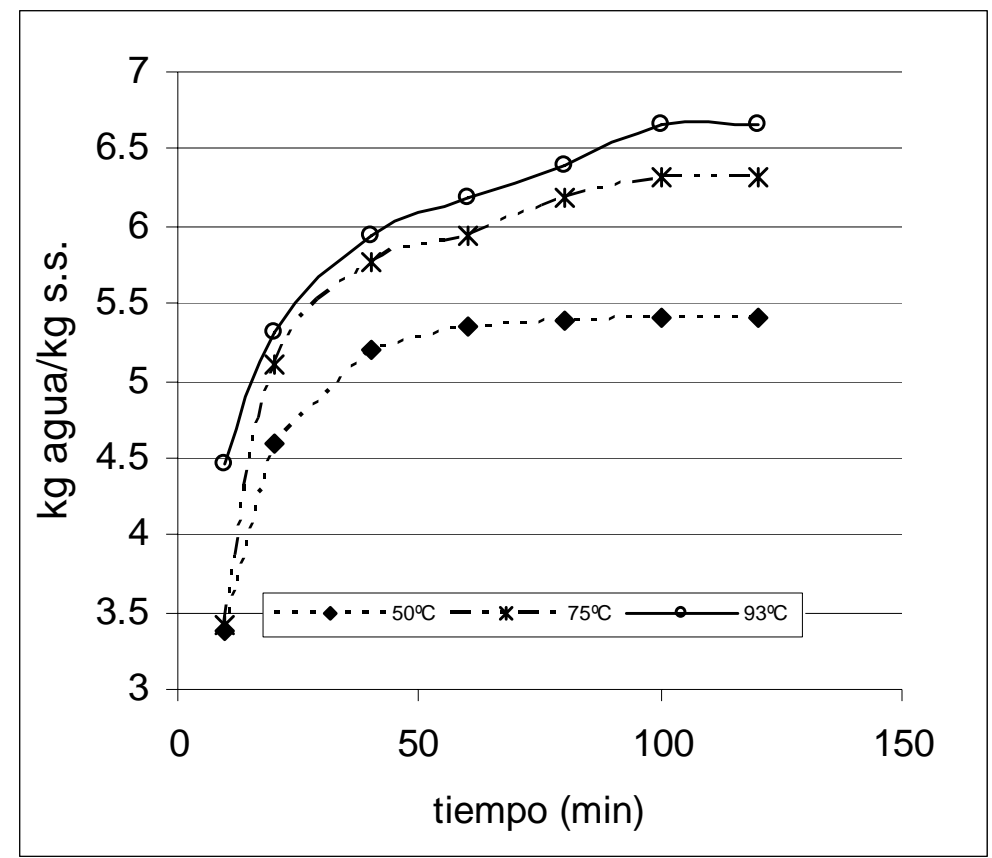

Fig. 1: Cinética de absorción de agua en zanahoria liofilizada.

El coeficiente correspondiente al ácido cítrico es el de mayor influencia para explicar el modelo de rehidratación de zanahorias liofilizadas $(p=0.001)$; además, en la explicación del modelo el coeficiente que explica la interacción $\mathrm{NaCl}$-ácido cítrico contribuirá a la explicación de las variaciones en el comportamiento de rehidratación, sin olvidar que en el modelo será también de consideración la interacción temperatura*ácido cítrico. Los coeficientes que no tendrán efecto significativo en el modelo serán el tiempo y la temperatura de rehidratación a las condiciones de límite inferior y superior a los que se desarrollo la experimentación.

De acuerdo con el análisis realizado y tomando en cuenta la $\mathrm{R}^{2}$, en el ajuste del modelo el termino del error debido a la variabilidad de los resultados fue de 0.383 , el de $R^{2}$-adj $=79.2 \%$, lo que valida el modelo cuadrático obtenido, que además es aceptable, si se considera que se trata de sistemas biológicos en donde la absorción de agua depende en gran medida de las características de la materia prima; así como, de las condiciones de proceso y conservación. En cuanto a los bloques y aleatoriedad del proceso, se establece un efecto de estos sobre los resultados obtenidos, $p=0.05$.

En la tabla 4, se muestran los resultados del análisis de varianza para los datos experimentales utilizados en el análisis de superficie de respuesta en función a los factores y sus interacciones, se observa que de acuerdo con los valores de $p=0.015$ para la regresión, los coeficientes obtenidos describen el modelo de comportamiento de la zanahoria durante su rehidratación; además, es necesario hacer énfasis en que los coeficientes cuadráticos no tienen efecto significativo en los resultados de la regresión para el modelo seleccionado $(p=0.714)$

Tabla 2: Constantes y coeficientes de difusión zanahoria.

\begin{tabular}{|c|c|c|c|c|}
\hline $\mathrm{T}\left({ }^{\circ} \mathrm{C}\right)$ & $\begin{array}{c}\mathrm{m} \\
\text { (kg de agua/kg } \\
\text { de s.s.) }\end{array}$ & $\begin{array}{c}k \\
\mathrm{~s}^{-1} \\
\times 10^{-4}\end{array}$ & $\begin{array}{c}\mathrm{D}_{\text {ew }} \\
\mathrm{m}^{2} / \mathrm{s} \\
\times 10^{-9}\end{array}$ & $\mathrm{R}^{2}$ \\
\hline 50 & 5.403 & 0.121 & 6.1 & 0.994 \\
\hline 75 & 6.314 & 7.53 & 4.05 & 0.958 \\
\hline 93 & 6.650 & 6.08 & 3.18 & 0.922 \\
\hline
\end{tabular}


Tabla 3: Coeficientes de Regresión de MSR

\begin{tabular}{|c|c|c|c|}
\hline Término & Coeficientes & $\mathrm{t}$ & $p$ \\
\hline Constante & 9.5382 & 3.99 & 0.003 \\
\hline Bloque 1 & -0.7710 & -7.38 & 0.000 \\
\hline Bloque 2 & 0.2208 & 2.11 & 0.061 \\
\hline Temperatura & -0.0145 & -0.32 & 0.750 \\
\hline Tiempo & -0.0552 & -0.29 & 0.772 \\
\hline $\mathrm{NaCl}$ & -0.889 & -1.39 & 0.194 \\
\hline Ac. Cítrico & -10.483 & -4.92 & 0.001 \\
\hline Temperatura $^{2}$ & 0.0001 & 0.52 & 0.610 \\
\hline Tiempo $^{2}$ & 0.0092 & 1.38 & 0.195 \\
\hline $\mathrm{NaCl}^{2}$ & 0.880 & 0.82 & 0.427 \\
\hline Citrico $^{2}$ & 0.9101 & 0.77 & 0.459 \\
\hline Temp*tiempo & -0.0021 & -1.35 & 0.204 \\
\hline Temp*NaCl & -0.0025 & -0.40 & 0.696 \\
\hline Temp*citrico & 0.0578 & 2.82 & 0.018 \\
\hline Tiempo*Nacl & 0.0053 & 0.172 & 0.867 \\
\hline Tiempo*citrico & 0.2169 & 2.119 & 0.060 \\
\hline $\mathrm{NaCl}^{*}$ citrico & 1.928 & 4.710 & 0.001 \\
\hline
\end{tabular}

Los términos que contribuyen en gran medida al comportamiento de la superficie de respuesta son los términos lineales y las interacciones en función a los valores de $p$, confirmando que los materiales alimenticios son entidades homogéneas en composición y estructura, la interacción entre los diversos factores, influye considerablemente en la rehidratación y comportamiento de los alimentos deshidratados (Oliveira e Hincano, 1999).

Con los datos obtenidos se establece que en el ácido cítrico debe de ser considerado como un parámetro importante en la formulación, cuando se utilicen como ingredientes productos deshidratados en la elaboración de sopas o productos instantáneos, puesto que provoca modificación en la integridad y capacidad de rehidratación del producto, sin olvidar que el $\mathrm{NaCl}$ y el ácido cítrico tienen un efecto sinérgico que influirá sobre las características y condiciones de rehidratación.

También de acuerdo a lo observado en las cinéticas de rehidratación, la temperatura juega un papel importante, recomendando que se manejen por encima de los $75^{\circ} \mathrm{C}$, con la finalidad de abatir el tiempo de rehidratación y por consecuencia las modificaciones texturales en la formulación de sopas y otros productos instantáneos, cumpliendo con el requisito de fácil preparación y utilización.

En la figura 2, se muestran los factores tiempo-temperatura, los que en forma independiente y como interacción tienen poco efecto significativo, aunque son variables que condicionan la rehidratación de la hortaliza desde el punto de vista cinético.

También se observa una ligera curvatura que de acuerdo al comportamiento y a la poco influencia de dicha interacción permite establecer que, en el caso de la rehidratación de zanahorias liofilizadas después de la adición de ácido cítrico y $\mathrm{NaCl}$, resulta que el tiempo no es una variable de influencia en el modelo; sin embargo, si tiene influencia en la cinética de rehidratación, así a mayor tiempo de rehidratación menor influencia de la temperatura en el proceso como se observa en la figura 2, no obstante este tiempo aumentará la rehidratación con la consecuente pérdida de calidad del producto por disolución de componentes. Un tiempo menor de rehidratación implicará un mayor incremento de temperatura para lograr incrementar la rehidratación. 
Tabla 4: Análisis de varianza para los modelos MSR.

\begin{tabular}{|l|c|c|c|}
\hline Fuente & DF & $F$ & $p$ \\
\hline Bloques & 2 & 28.91 & 0.000 \\
\hline Regresión & 14 & 4.08 & 0.015 \\
\hline Lineal & 4 & 6.61 & 0.007 \\
\hline Cuadrático & 4 & 0.53 & 0.714 \\
\hline Interacciones & 6 & 6.11 & 0.006 \\
\hline
\end{tabular}

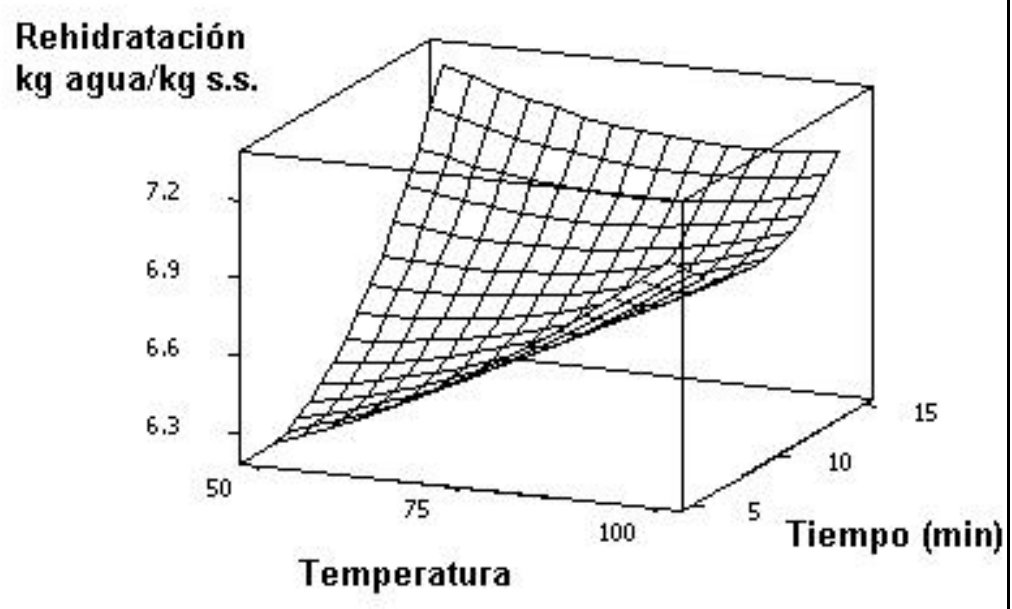

Fig. 2: Efecto tiempo vs temperatura sobre la rehidratación

En la figura 3, se representa el comportamiento de superficie de respuesta para la temperatura en interacción con el ácido cítrico, la que de acuerdo con la tabla 3, resulta ser una de las interacciones con mayor efecto significativo en el comportamiento de rehidratación de la zanahoria liofilizada, observándose que a baja concentración de ácido cítrico se presenta una mayor rehidratación sin efecto significativo de la temperatura; sin embargo, la temperatura sin influye en la rehidratación al aumentar el contenido de ácido cítrico, siendo menor a altas concentraciones.

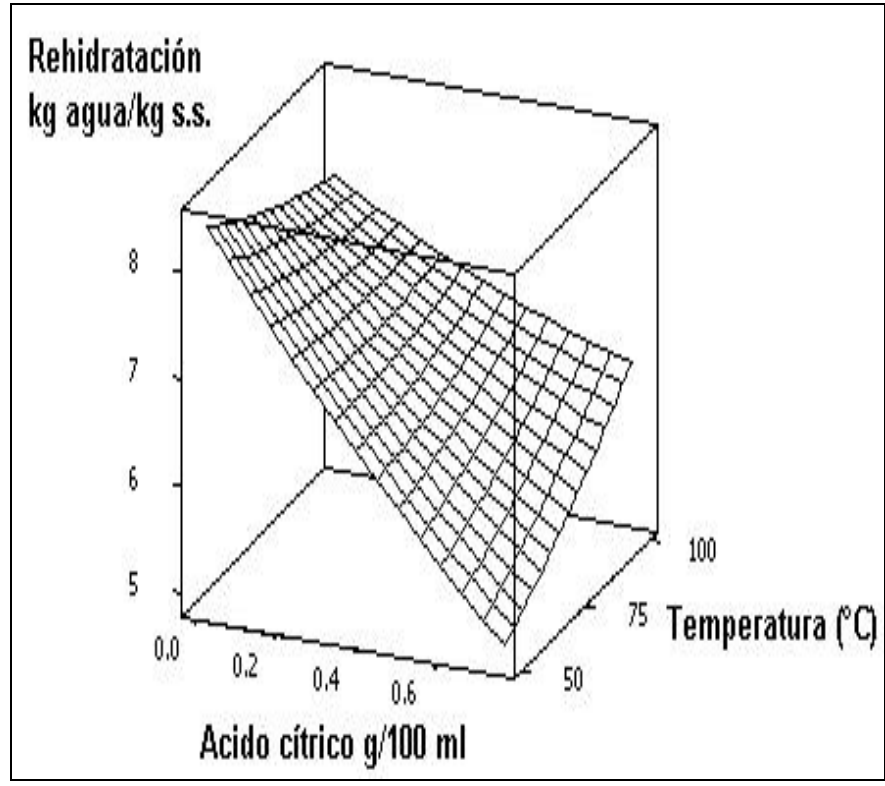

Fig. 3: Interacción acido cítrico vs temperatura.

En la figura 4, se muestra el comportamiento de la rehidratación de zanahoria liofilizada por efecto de la interacción entre el ácido cítrico y $\mathrm{NaCl}$, por abajo de una concentración de $0.4 \mathrm{~g}$ de ácido cítrico/100 ml, hay un aumento de la rehidratación hasta por encima de $7.5 \mathrm{~kg}$ agua/kg de s.s., con una mayor respuesta cuanto menor es la concentración de $\mathrm{NaCl}$. Conforme aumenta el contenido de 
$\mathrm{NaCl}$ y a concentraciones por encima de $0.35 \mathrm{~g}$ de ácido cítrico/100 ml hay una disminución de la rehidratación hasta $5.23 \mathrm{~kg}$ de agua/kg s.s., que indica un comportamiento sinérgico entre ambos.

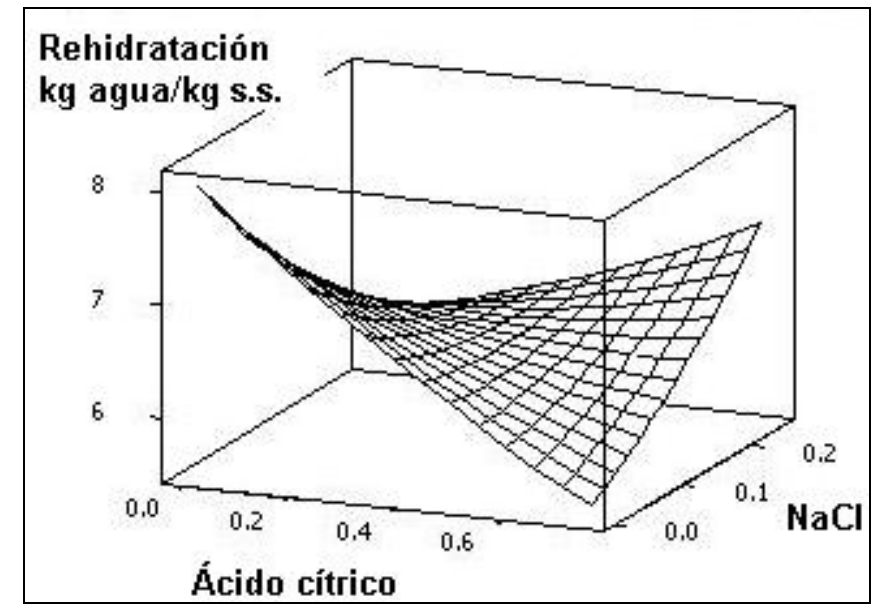

Fig. 4: Interacción acido cítrico vs $\mathrm{NaCl}$.

De acuerdo con los valores reportados para rehidratación de vegetales que oscilan entre 6 a $10.5 \mathrm{~kg}$ de agua/kg s.s., (Femenia et al., 2000; Krokida, 2003), los análisis de optimización del modelo matemático se realizaron considerando en promedio un valor de rehidratación mínimo de $6.5 \mathrm{~kg}$ de agua/kg de s.s. y un máximo de $7.5 \mathrm{~kg}$ de agua $/ \mathrm{kg}$ s.s.

En la tabla 5 se muestran los resultados correspondientes a la optimización del modelo en función a las variables y límites involucrados en el diseño experimental. De esta se tiene que para obtener los valores pre-establecidos, la variable de mayor efecto es la concentración de ácido cítrico ya que oscila desde 0 a 0.636 dependiendo de la rehidratación en estudio, lo cual concuerda con el análisis realizado anteriormente, en función al efecto de las variables involucradas.

Tabla 5: Optimización rehidratación de zanahoria liofilizada.

\begin{tabular}{|l|c|c|c|}
\hline Factor/ respuesta & \multicolumn{3}{|c|}{ Valores factores/respuesta } \\
\hline Temperatura $\left({ }^{\circ} \mathrm{C}\right)$ & 75 & 75 & 75 \\
\hline Tiempo (min) & 12.38 & 10 & 10 \\
\hline NaCl g/100 ml & 0.250 & 0.250 & 0.250 \\
\hline Ácido cítrico g/100 ml & 0 & 0.307 & 0.636 \\
\hline $\begin{array}{l}\text { Rehidratación kg de } \\
\text { agua/kg s.s. }\end{array}$ & 6 & 6.5 & 7.0 \\
\hline
\end{tabular}

\section{CONCLUSIONES}

Para el caso de la cinética de rehidratación agua-zanahoria liofilizada, temperaturas entre 75 y $93^{\circ} \mathrm{C}$, no muestran diferencia significativa con constantes cinéticas que varían en un $19.74 \%$ y un valor promedio de absorción de agua de $6.48 \mathrm{~kg}$ de agua/kg de s.s.

Los coeficientes de difusión $\left(D_{e w}\right)$, son menores conforme se incrementa la temperatura, lo que implica que existe una menor resistencia a la absorción de agua y pese a que el valor a $75^{\circ} \mathrm{C}$ es de $4.05 \mathrm{y}$ el de $93^{\circ} \mathrm{C}$ de $3.18 \mathrm{~m}^{2} / \mathrm{s}$, es necesario tomar en consideración la variabilidad de otros aspectos de calidad del producto a rehidratar.

Es importante resaltar que en relación con la cinética agua-zanahoria, la adición de ácido cítrico y $\mathrm{NaCl}$, influyen considerablemente en el comportamiento de la rehidratación, incrementándose hasta 
$7.89 \mathrm{~kg}$ de agua $/ \mathrm{kg}$ de s.s. en los niveles establecidos para las variables y que están en función de los hábitos de consumo.

La temperatura tiene un efecto significativo cuando interactúa con el ácido cítrico, por lo que debe ser examinada cuidadosamente en la elaboración de productos instantáneos que contienen vegetales deshidratados, ya que al aumentar el tiempo de rehidratación también aumentara la capacidad de rehidratación independientemente de la temperatura, lo que a su vez modificará la calidad del producto, ya que al aumentar el tiempo traerá como consecuencia la disolución de componentes de la zanahoria.

En relación al modelo de predicción obtenido para la MSR, la variable de mayor influencia fue la variación en la concentración de ácido cítrico, aspecto importante ya que México es una de las regiones del mundo donde más se utiliza el limón para aderezar los alimentos, que contribuye en gran medida a los cambios texturales y de pérdida de solutos durante la preparación de comida rápida que contenga zanahoria, ya que conforme se incrementa la concentración disminuye considerablemente la capacidad de rehidratación con valores de hasta $4.5 \mathrm{~kg}$ agua $/ \mathrm{kg}$ s.s.

Existe un efecto sinérgico entre la concentración de ácido cítrico y $\mathrm{NaCl}$ a concentraciones entre $0.325-0.42 \mathrm{~g} / 100 \mathrm{ml}$ y concentraciones de entre 0.125 y $0.15 \mathrm{~g} / 100 \mathrm{ml}$ de $\mathrm{NaCl}$, ya que bajo estas condiciones la rehidratación es de $7 \mathrm{~kg}$ agua $/ \mathrm{kg}$ de s.s., modificándose considerablemente en los extremos y salvo las concentraciones menores, existe una disminución en la capacidad de rehidratación de zanahoria.

\section{NOMENCLATURA}

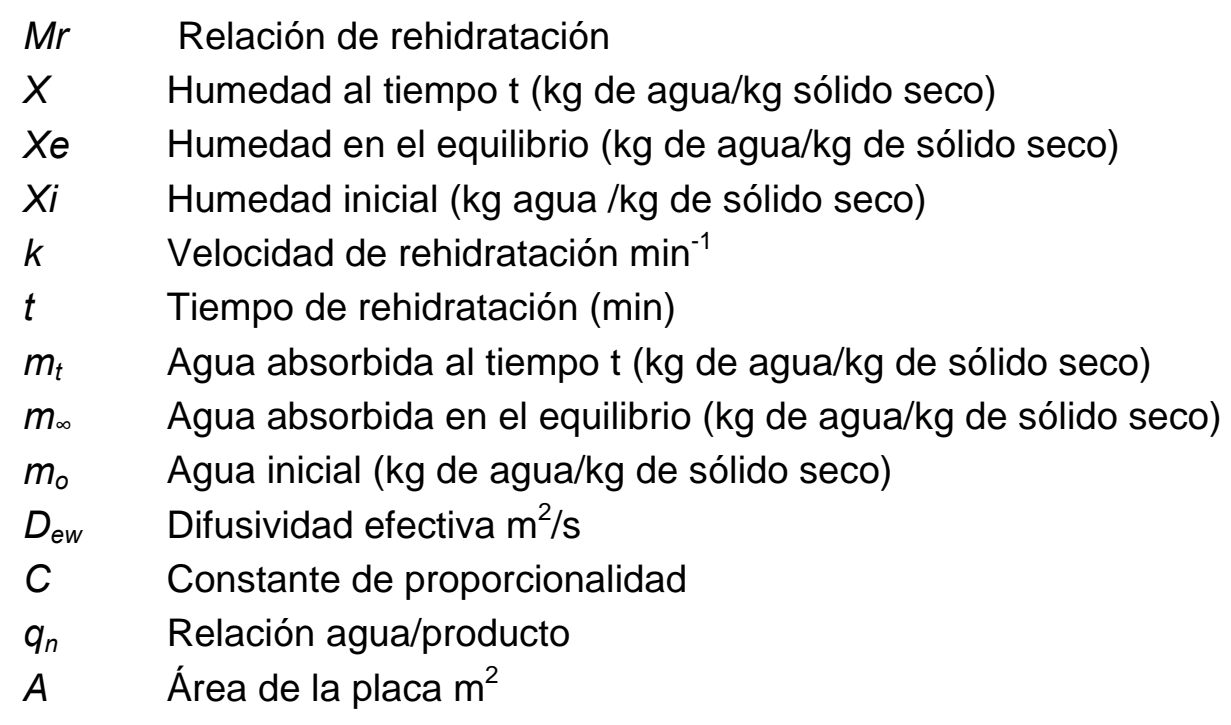

\section{REFERENCIAS}

AOAC; "Official methods of analysis of AOAC International" / William Horwitz, Ed. Association of Official Analytical Chemists (2005)

AACC; "Approved Methods of the American Association of Cereal Chemists". (1983).

Barbosa, G.; “Deshidratación de los Alimentos”. Editorial Acribia. Zaragoza, España. 203-232 (2000).

Femenia, A. y otros cuatro autores; "Efect of rehydration temperature on the cell wall components of broccoli (Brassica oleracea L. Var. italica) plant tissues” J. Food Eng. 46: 157-163 (2000).

Horwitz, W.; "Official Methods of Analyisis". 17 ${ }^{\text {th }}$ Edition. Vol.II. Cap. 42, 1-14p. AOAC International. Maryland, USA. (2000). 
Krokida M.K. y B. Maroulis; "Effect of Freeze-drying Conditions on Shrinkage and Porosity of Dehydrated Agricultural Products”. Journal of Food Engineering. 35: 369-380 (1998).

Krokida, M.K., V.T. Karathanos, Z.B. Maroulis y D. Marinos-Kouris; "Rehydration kinetics of dehydrated products". Journal of Food Engineering.57: 1-7 (2003).

Lee T.K., M. Farid y S.K. Nguang; "The mathematical modelling of the rehydration characteristics of fruits" J. Food Eng 76: 16-23 (2006).

Litvin S. y J. Milt J.; "Dehydration of Carrots by a Combination of Freeze Drying, Microwave Heating and Air or Vacuum Drying". Food Engineering. 36: 103-111. (1997).

Madamba. P.S.; "The Response Surface Methodology: An Application to Optimize Dehydration Operations of Selected Agricultural Crops". Institute of Agricultural Engineering, University of the Philippines. 584-592 (2002).

Mafart, P.; "Ingeniería Industrial Alimentaria. Procesos Físicos de Conservación”. 1: 242-252. Editorial Acribia. Zaragoza, España. (1994).

Marabi, A., U. Thieme, M. Jacobson e I.S. Saguy; "Influence of drying method and rehydration time on sensory evaluation of rehydrated carrot particulates. Journal of Food Engineering. 30:1-7. (2004).

Rastogi, N.K., Nayak C.A. \& Raghavarao M. S.. "Influence of osmotic pre-treatments on rehydration characteristics of carrots". Journal of Food Engineering. 65:287-292. (2004).

Wang, J. y Y.S. Xi; "Drying characteristics \& drying quality of carrot using a two-stage microwave process. Journal of food Engineering". 68:505-511 (2005).

Zambrano M.L., N.T. Gallardo y P.R. Meléndez; "Propiedades funcionales y metodología para su evaluación en fibra dietética". En: Fibra dietética en Iberoamérica, J.M. Lajolo pp195-209 Valencia, España (2001). 